\title{
Erratum to: Identification of two novel rabbit hemorrhagic disease virus (RHDV) B cell epitopes and evaluation of its immunoprotection against RHDV
}

\author{
Kong DeSheng ${ }^{1} \cdot$ Liu HuaiRan ${ }^{1} \cdot$ Liu JiaSen $^{1} \cdot$ Yu Zuo ${ }^{1} \cdot J_{i a n g}$ Qian ${ }^{1}$. \\ Guo DongChun ${ }^{1} \cdot$ Hu XiaoLiang ${ }^{1}$ - Wang FengJie ${ }^{1}$ - Huang QianQian ${ }^{1} \cdot$ Qu LianDong ${ }^{1}$
}

Published online: 22 May 2015

(C) Springer-Verlag Berlin Heidelberg 2015

Erratum to: Appl Microbiol Biotechnol (2015)

DOI 10.1007/s00253-015-6571-8

The original version of this article inadvertently contains mistake. Figures 3, 4 and 5 images and legends have been interchanged. Correct presentation given below:

Insert Figures 3, 4 and 5 and it's corresponding legends.

\section{Liu JiaSen}

neauljs@163.com

Qu LianDong

qld@hvri.ac.cn

Kong DeSheng

guwangdaben@163.com

Liu HuaiRan

huairan@163.com

Yu Zuo

yuzuosy@163.com

Jiang Qian

jiangqian623@sina.com.cn
Guo DongChun

gdongchun@126.com

$\mathrm{Hu}$ XiaoLiang

liang679@163.com

Wang FengJie

flyinggirl.1988@163.com

Huang QianQian

huangqq0521@126.com

Zoonosis of Natural Foci, State Key Laboratory of Veterinary, Biotechnology, Harbin Vet Res Institute, Chinese Academy of Agricultural Sciences, 427 Maduan Street, Nangang District, Harbin 150001, People's Republic of China 
a

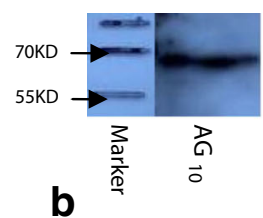

RHVV-VP60 AAII21587.1 VTIGLSLNNHSSALIPGQFFYWQLTFASGFIEIGLSWDGYYAGTGASTTLIDLTELIDVRPVG

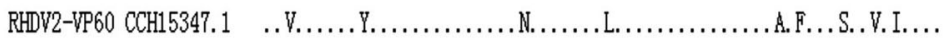

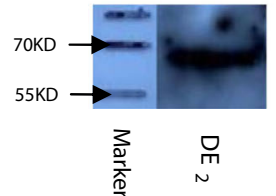

RHDY-YP60 AAl21587.1 FYhQLTFASGFIETGLSYDGYFYAGTGASTTLIDLTELIDYRYGPRPSRSTL

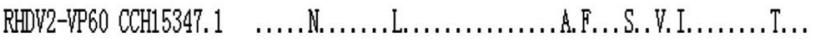

Fig. 3 Reactivity of recombinant VP60 expressed by Bac-to-Bac Baculovirus Expression System with $\mathrm{mAbs} \mathrm{AG}_{10}$ (a) and $\mathrm{DE}_{2}$ (b) by Western blotting. Blots were developed using a Super Enhanced chemiluminescence detection kit. The protein bands were visualized after exposure of the membranes to Kodak X-ray film. The amino acid sequences corresponding to the regions encompassing the two epitopes from RHDV-TP strain and RHDV2 were aligned. The epitope recognized by $\mathrm{AG}_{10}$ had nine amino acids substitutions (a) and the epitope recognized by $\mathrm{DE}_{2}$ had eight (b)
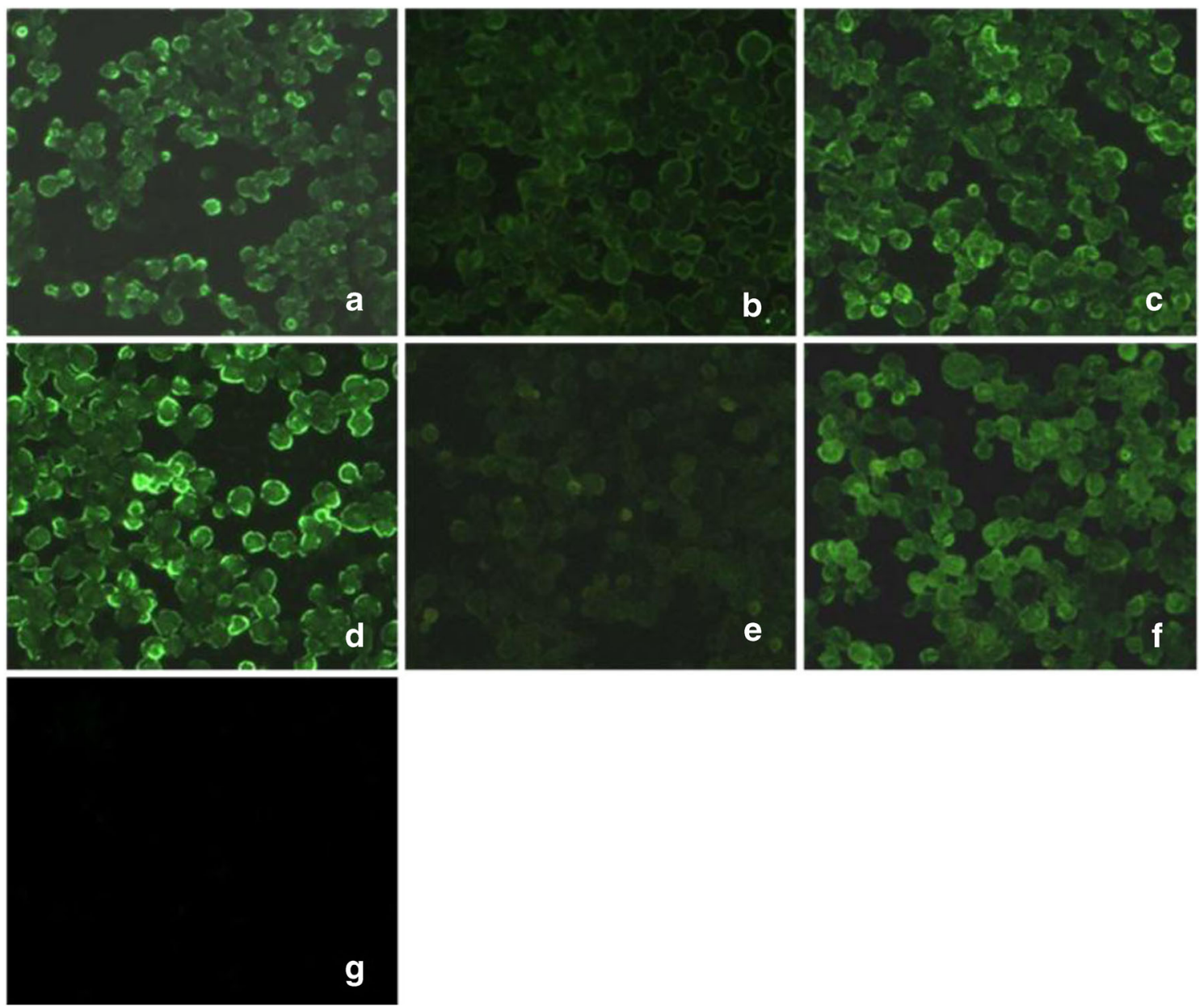

Fig. 4 Immunofluorescence analysis of reactivity between RHDV mAbs and Sf9 cells expressing RHDV VP60 protein. a-f Cells infected with recombinant baculoviruses reacted with $\mathrm{mAbs}\left(\mathrm{AD}_{4}, \mathrm{BC}_{9}, \mathrm{DE}_{2}, \mathrm{AG}_{10}, \mathrm{BE}_{8}, \mathrm{BH}_{3}\right)$ and a negative mouse serum control $(\mathrm{g})$ 
a
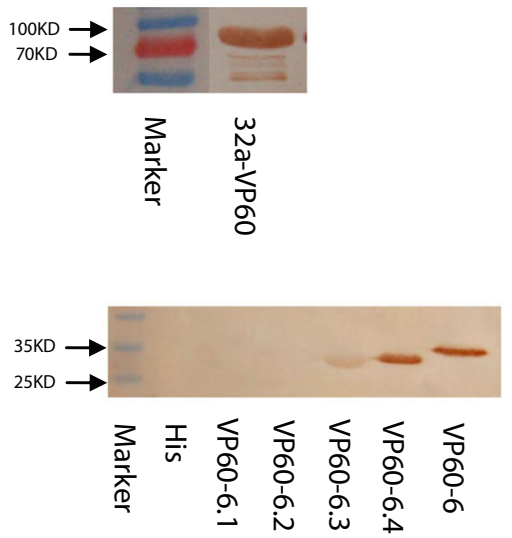
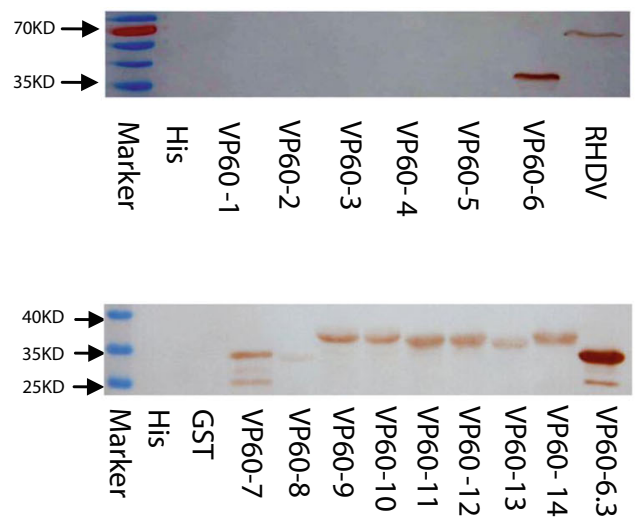

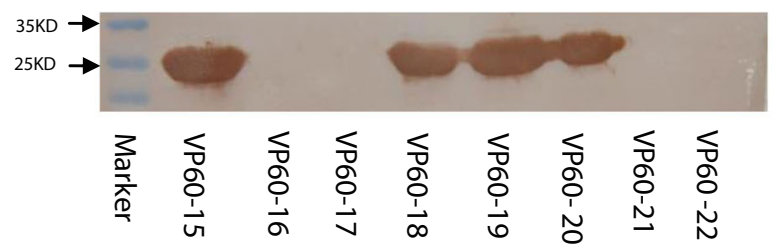

b
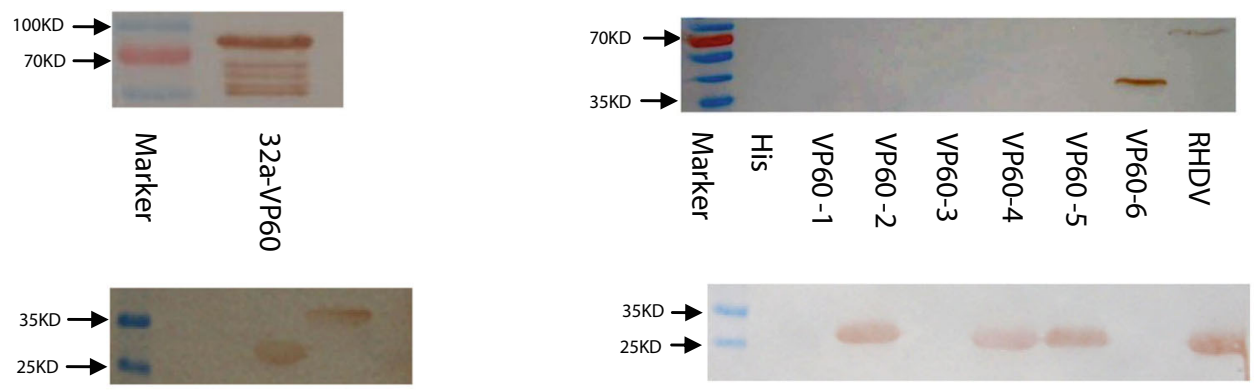

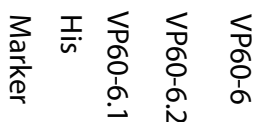

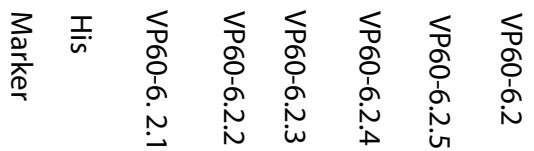

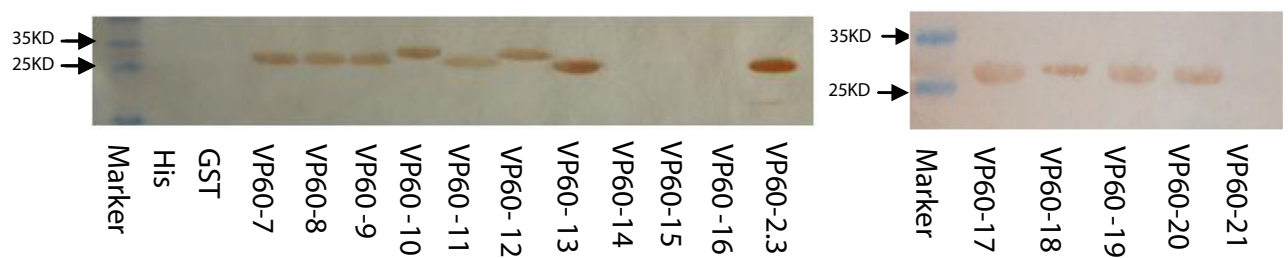

Fig. 5 Fine locations of $\mathrm{mAb}_{A G_{10}}$ and $\mathrm{DE}_{2}$ epitopes. Reactivities of $\mathrm{AG}_{10}$ (a) and $\mathrm{DE}_{2}$ (b) with truncated recombinant VP60 protein by Western blotting. Names of the truncated proteins are shown in Table 1. His and GST were used as negative controls, and RHDV virus and the shortest recombinant VP60 of every truncate were used as positive controls. VP60 was first truncated into 6 segments and $\mathrm{mAb} \mathrm{AG}_{10}$ and
$\mathrm{DE}_{2}$ were both recognized segment 6 (VP60-6). Then the next truncations were all related to peptide VP60-6. VP60-15 and VP60-20 determined the $\mathrm{N}$-terminal and $\mathrm{C}$-terminal amino acid scope of $\mathrm{AG}_{10}$ recognition sequence in a, whereas the scope of $\mathrm{DE}_{2}$ recognition sequence was determined by VP60-13 and VP60-20 in $b$ 